\title{
APOLLINAIRE ET L'AVANT-GARDE CATALANE: COINCIDENCES ET INFLUENCES SUR JUNOY ET SALVAT-PAPASSEIT
}

\author{
POR \\ J. IGNACIO VELAZQUEL
}

L'oeuvre d'Apollinaire est introduite en Espagne, du vivant de l'auteur, d'une façon graduelle et hétérogène '. C'cst, d'abord, à travers les traductions et les références de première ou de seconde source et, ensuite, en rapport avec ce que le mythe culturcl parisien représente pour les avant-gardes européennes du dćbut du siècle, qu'une image d'Apollinaire se constitue peu à peu. Image incertaine, elle-même n'aurait sans doute pas déplu à notre poète.

Il commence à se faire connaître en Espagne avec un léger retard par rapport au futurisme, et il faudra encore attendre un certain temps pour que les différences qui séparent le poète du mouvement s'établissent nettement. Tout se passe comme si la théorie de al'état d'esprit» l'emportail sur les écoles de façon à équilibrer la multiplicité et l'éparpillenent de celles-ci par la notion globale d'«avant-garden. Il se peut que la difirsion de cut «esprit moderne» en soit sortie bénéficiée. En tout état de causc, pour les jeunes poètes espagnols des années 10, la connaissance de la poétique apollinarienne comporte celle des mouvements futuriste et cubiste, parmi d'autres.

Quelles sont les données qui encadrcnt l'arrivée de ces avant-gardes en Espagne? D'une part, le rốle jouế par Ramón Gómez de la Serna (celui-là même que Cocteau nomme le Max Jacob espagnol, alors que pour Robert Delaunay, il était l'Apollinaire espagnol), à travers la revue poétique fondée

\footnotetext{
' On peut prendte pour point de dépari les queluues arcicles consacrés aux rapports entre le poìte ec l'Espagne ct, en particulier, ceex de W. BoHN: "Guillałme Apollinaire: Homage From Catalonian, in Symporinm, vol. 33, n." 2, 1979, pp. 101-117; de R. WakNik: "Apollinaire, le Ponural er l'Espagnen, in Bulletin des Etudes Portugaises, n."22, 1959-60, er de M. DEcaudin: "Ápollinaire outre-Pyrénécss, in Revne des Lettres Mindemer, n," 123-126, ainsi gue les recherches poussetes dans le donaine calitaria par Pierre Caizcrgucs.
} 
par son père, «Prometeo», quc publie en 1909 —la même année qu'en France et en Italie- le Manifeste Futuriste de Marineti, qui publie en 1910, dans le $12 .{ }^{\circ} 10$, la Proclama futurista a los españoles, signéc «Tristán»-pseudonyme de Gómez de la Serna, et, dans le n. ${ }^{\circ} 20$, une seconde Proclama Futuriststa, signée cette fois-ci par Marinetti lui-mềme.

1912 est une année importante pour le cubisme en Espagne 2 . On présente unc première exposition cubiste à Barcelone, et J. M." Junoy publie, à Madrid, «Artc y Artistas», où il foumit une analyse du mouvement. Madrid devra attendre jusqu'cn 1915 pour apprécier les cubistes du «Salón del Arte Moderno», près de Pombo, le café où règne Gómez de la Serna et que Marie Laurencin, les époux Delaunay ct Picasso, parmi d'autres, vont fréquenter.

C'est la période de la guerre er un certain nombre de refugiés français convergent vers l'Espagne, pays qui reste à l'écart du conflit. Madrid et Barcelone sont les lieux de rencontre. A Barcelone, les Galeries Dalmau organiscnt en décembre 1915 et janvier 1916 une exposition Van Dongen, et en novembre-décembre 1916 une autre de Gleizes. A leur suire, Picabia, Arthur Cravan, Canudo, ctc., qui font paraître à partir de janvier 1917 le 4 numéros en français - 16 pages en tout- de «391» où l'on trouve, en plus, les signatures de Max Jacob et Ribemont-Dessaignes. La même année, Parade est représentée à Madrid et à Barcelone et, dans la revue «Troços" on annonce une éventuelle première des Mamelles de Tiressias ${ }^{3}$, adaptée en catalan, à Barcelone.

Défầ vers 1918 les tendences d'avant-garde - les «ultraístas» surtout où l'on rencontre à nouveau le couple Delaunay, les «vibracionistas», etc.ont pris une certaine envergure, s'appuyant sur les revues poétiques: «Prometeo», «Perseo», «Troços», «Ultra», «Un enemic del Poble», "Grecia», à Seville, «Arc-Voltaîc», qui ne connaîtra qu'urn seul numéro, tout comme «Proa», et bien d'autres. Et en 1920 les premicrs noms espagnols apparaissent dans le Bulletin 12. 5 de Dada: Dalmau, Junoy, Carsinos-Assens, Guillermo de Torre et Lasso de la Vega. Et encore, dans la meme année, une exposition \&Art Francés d'Avantguardias réunit à Barcelone les noms de Léger, Marie laurencin, Braque, Dufy, Miró, Picasso, Derain, Gleízcs, Metzinger, Matisse, Severini, Vlaminck, Van Dongen et d'autres..

$$
\stackrel{*}{*} *
$$

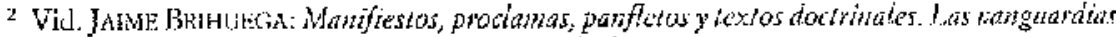
artisticas en España, 1910-1931, Ed. Cúledra, Madrid, 1979.

3 "Una adaptació catalana del drama sobrercaliste d'Apoltivaire Les Mameles de Tirésias és anunciada per Trossos a sos llegidors. Lina representació de la mareisa obra procurara donar-sc cn sessió privada sí un ajut i una collatoració li son propicisw, Trosson, u." 5 . Barcelone, avril 1918̂, p. 8. La nutson d'édition Leeeradura, de Barcelone, a publié tes nunétos tacsimilés de (1n enewtic del Poble, Proa at Axc Volitite (1976), et Troços et 391 (1577) 
Dans ce contexte et pendant ces années, la figurc d'Apullinaire ne passe pas inaperçue. Il y a eu des voyages et des contacts dans lesquels Picasso a joué un tole déterminant. Huidobro parlera de lui à Madrid et autant les Delaunay que Maric Laurencin fréquentent Gómez de la Serna qui, lui, ne le connaîtra que vers la fin de sa vie mais restera fortement marqué par le poète français. Il est connu à travers les rapports épistolaires et, surtout, à travers ses oeuvres dont un certain nombre de contes, de poèmes ou d'écrits théoriques commencent à être traduits. Apollinaire correspond avec Folyuera, avec Junoy, avec Francesc Carbonell... Junoy publie, et c'est bien connu, en 1920, comme préface des Pocmes $i$ Calligrames la «Carta-Prefaci» qu'Apollinaire lui a adressée en le félicitant pour ce chef-d'oeuvre: "Guyne. mer». Cansinos-Assens, Guillermo de Torre, Lasso de la Vega et Diez-Canedo, entre autres, ke traduissent.

En 19.17, les cinq numéros de «Troços» parlent de lui. Et, en 1918, dans la revue «España», paraît la traduction de «La desaparición de Honorato Subrac» qui sera par la suite reptise par la revue "Grecia» en plus d'un article sur la «Nowvelle Peinture» traduit des Peinires Cubistes, et de «Le Chant d'Amour»*. Les recherches de Pierre Caizergues prowwent qu' $\alpha$ lberia» et «La Publicidad» se sont fort occupées de luí. \&La Publicidad», Iors de sa mort, va parler de lui pendant plusieurs jours, mais elle avait déjà publié en juillet 1918 - teil qu'on l'a déjà établi lors d'un précédant Colloque et publié dans «Les Lettres Modernes»- un interview de J. Pérez-Jorba avec Apollinaire intitulé «Paseando con Apollinaire», «Cosmópolis» publie, début 1919, Ia traduction de L'Esprit Nowveatu et les Poctes; "Cervantes» celle du Guide et «Grecia» celles de plusieurs poèmes. Peu après, c'cst à

\footnotetext{
‘ Voir, dans España, n."189, novembre 1918, l'êloge funëbre de Guillersto de Torre, repris dans Cervantes (levier 1919). La desaparicion de lomorato Subrac, traduite par Diez-Canedo qui verra anssi le jour dans Grecia [20 jullet 1919), en plus d’une ćrude er La Nireva Pintura. traduite des Peintres Cubistes par Dícz-Canedo, ainsi que El Cmio de Anor. Voir aussi Cosmó. polis, ns." 1, janvier 1919: «Un estzdio póstumo de Apollinaite. El espiritu nuevo y los poetas», op. 17-28, traduit probablemen par Cansinos Asscns: Ceroawts, férier 1919: «Novisina litcratura francesa, El Guía. Una aventura del Barón cl'Ormesan, por Guillemo Apollinairen (sic), pp. 89-95, traduis par Guilletmo de 'Torre; Grecia, n." 11, 15 mars 1919: «La Novisima Literatura Francestw, avec une raduction de $L e$ Chant "Amom, par Cansinos-Assers; Cervanres, mars 1919: «Novisima literaura francesa. Solre Gujisermo Apollionarem, pp. 70-76; Grecia, 12." 12, wril 1919: "Antes del cinema", traduit par Cansinos- 1 ssens et repris dans Ceriantes (mai 1919), pp. 90-93, de mĉme que «Cohere-señal» et «Océano de la tierra»; Grecida, no" 20 . 30 juin 1919: «Guillaume Apollinaire», par 13. Dinz-Cantedo, et no" 22, 20 jujllet 1919: "La desaparictión de H. Subrac*, n. 24: «Guiliaume Apollinairc. Salomén, rraduit par Diez-Cancdo,

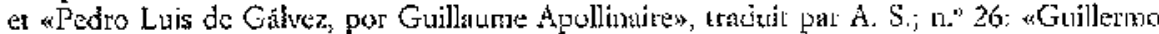

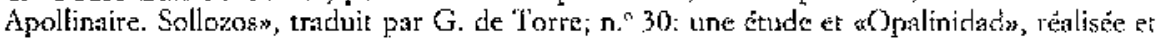
traduite par G. de Torre; n." 36, 20 décembre 1919: «Hacia cl \$ur», raduir par G. de Torre: Whira, n." 4, I mars 1921: «69666.. 6 9... Jos inversos 6 y 9m, traduit par R. Lasso de la Vega; n." 19, I décembre 1921: «Un poema discucido de Apollinairem, par Guillermo de Torre, etc.
} 
nouveau «Cervantes» qui publie une étude sur Apollinaire de Jean Royère, traduite par Cansinos-Assens. De leur côté et pendant toute l'année 1919, «Grecia" et "Cervantes» font apparaittre constanment de nombreuses traductions de poèmes, de contes et jusqu'ì l'sanecdotiquex qui se rapporte à Pedro Luis de Gálvez. Ce sera, par la suile, la revue «Ullra» qui prend la relève el peu après on troive les références des premiceres traductions aujourd'hui introuvablow, de El teatro italiano et Relatos, ainsi que la première version, on 1924, du Puèté Assassiné, préfacé par Gómez de la Serna sous le titre « $\Lambda$ pollinaire, el precursor», de même que la première édition française de It $y$ a, en 1925.

Il ne s'agit pas de dresser un inventaire complet, mais uniquement de montrer, par les preuves, l'intérêt éveillé par le poète dans l'avant-garde espagnole, et le degré de connaissance que celle-ci a d'Apollinaire. Encore un exemple: lorsque Gómez de la Serna publie Ismos, en 1931, où il passe en revue les différentes tendances du XX siècle, le premier chapitre s'intitule «Apollinerismo» et il y fait unc étude du poète. Ensuite, il y anta « Picassismo» et «Futurismo», tt puis bien d'autres. Pourquei avoir placé Apollinaire le premier? «Les -ismes de ce livre n'ont pas été ordonnés au hasard», explique-t-il. Et il continue: «Como Apollinaire es un poco la primera letra capitular de todo lo que sucede, va en cabeza su biografías ${ }^{5}$. Et ensuite, il traduit la préface de $I t y$ a, dans l'édition de La Phalange.

Disons donc qu'avant sa mort, Apollinaite se trouve introduit dans les cercles - qu'il commence mêmè à dépasser, fort des son prestige en tant que «chef de file» des poètes français- de l'avant-garde espagnole. On connaît sa prose: Le Poète Assassitté et plusieurs contes traduits de L'Hérésiarque et Cie., mais on le lit en français. On connaît aussi Alcools et, en Catalogne surtout, on aime beaucoup Calligrammes. On dispose de téférences concer. nant Les Mamelles et on prend pour point de départ théorique ses écrits concernant le cubisme et $l^{\prime}$ «Esprit Nouveau». Des tas de références, plus ou moins directes, augmentent son prestige. D'où le fait que cette floraison de revues poétiques qui apparaissent dans les années 10 s'accrochent à lui -jusqu'à, à peu près, les ammées 30-, et se toument vets son ouvte, mulipliant les traductions, les études ou, simplement, apportant des nouvelles sur lui. Sa mort ne fera qu'accrôtre l'intérêt évejllé: dans le revues, on entreprend ce travail de diffusion d'une façon plus systématique et simultanée. Tâche à laquelle participent les poètes d'avant-garde les plus importants.

Mais cela ne doit pas pour autant faire croire que le poète ait toujours èté bien accueilli et bien compris. Sans atteindre les agressions critiques d'un Duhamel ou d'un Vaché, il sera aussi contesté en Espagne. Par Espina

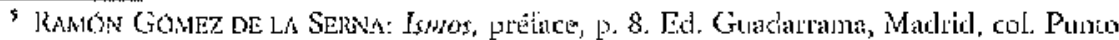
Oncga, 1975. 
Gracia, en 1919, dans «L'Instant» par exemple, quand il veut démontrer qu'aucun poète espagnol «ultraísta» n'est à la hateur ad'un Reverdy, d'un Cendrars ou d'un Apollinairc-mu'est-ce qu'ils ont pu nous casser les pieds avec ce dernier-, même si tous les trois ne sont que des poètes de seconde on de troisième calégoric̀. Ou par Foix lui-même, le second directeur de «Troços», dans «L'Anic de les Arts», on 1927, lorsqu'il analyse «ce que c'est que l'avant-garde» pour en condure que «Apollinaire et ses plagiaires ont été les poètes les moins avant-yardistes de toute luur tubulente génération». Ou lorsque, la même année, Gasch cite un plus que douteux propos d'Apollinaire, dans «La Gacta Literaria", d'après lequel les seuls tableaux valables de Chirico seraient ceux que le peintre avait réalisés lors de sa maladie estomacale. N'empêche qu'à la même époque, Lluis Montanyá écrit, dans «L'Amic de les Arts»: "Révolutionnaires, les cubistes l'ont été, les cubistes littéraires dont Apollinaire est resté le guide très senwiblex.

Qu'il était cotnu en Espagne est donc indéniable. Qu'on se forçait de bien le connaître, est certain. Mais il faut tenir compte aussí que l'adhésion générale n'était pas uranime et qu'il y avait aussi des voix pour le contester.

Voyons maintenant de plus près ce qui se passe en Catalogne. Les poètes s'y trouvent très actifs dès le dêbut du siècle. Ils participent des inquiétudes poćtiques européennes auxquelles ils se montrent très réceptifs. On possède une preuve intéressante et peu connue dans le calligramme réalisé en 1905 par Rafael Nogueras Oller pour Les Teneses, qui porte pour titre «Una essem. Il est certain pourtant que ces courants avant-gardistes buttent $\mathrm{d}^{2} \mathrm{une}$ part avec le snoucentismon et d'autre part avec tout le contenu idéologique et nationaliste dont nombre de poètes se font l'écho. Et que l'affrontement de ces tendances leur fait perdre beaucoup de forces et de moyeris d'cxpression. Mais il r'en est pas muins certain que «cubisme poétique» et «Esprit Nouveat»s se manifestent avec vigueur. On a dćjà vu l'importance de l'exposition cubiste de 1912, dans les Galeries Dalmat auxquelles Junoy est très lié. Quels sont les noms qu'on y trouve? Ceux de Gris, Duchamp, Gleizes, Metzinger, Agero et, aussi, Marie Laurencin. Junoy soutient le cubisme dans Arte y Artistas, mais aussi dans les pases de la revue, fondée la mëme année, "Correo de las Letras y de las Artes». Mais qui dit «cubisme» à cette époque-là, dit surtout «avant-gardes en Catalogne. Le premier contact avec le futurisinc remonte, au moins, à 1904, lorsque Gabriel Alomar fait à Barcelone une conférence intitulée «Le Futurisme», introduisant l'école en Espagne cinq années avant le Manifeste et influcnçant Marinetti lui-même, dans les aspects idéologiques du mouvenent: ${ }^{\circ}$.

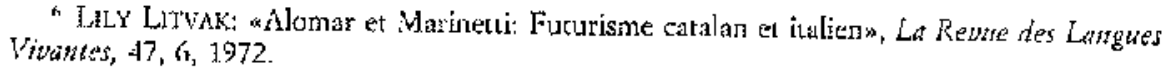


En échange, la Grandc Guerre apporte de nouvelles possibilités d'expression à l'avant-garde françaisc ì Barcelone. Il y a l'sxemple peu connu de la revue alberias dont la création doit beaucoup au Consulat Français, et qui sert de porte-parole au partisans de la France. Junoy y participe très activement et la revue joue en rôle essenticl dans la diffusion de la culture française. On y trouve des articles sur Claudel, sur Romain Rolland, etc., etc. Itt dans Ie n." 191, le 14 décembre 1918, Francesc Carbonell parle de la mort d'Apollinaire dans l'article «Les revues» en y insérant, en grise d'hommage, le fragment d'une lettre que le poère lui a adressée, déjá merntionnée par M. Décaudin dars «Apollinaire outre-Pyrénées»:

«La guerra lo había herido ya dos veces, pero se habia salvado de la muerte. La noticia de su fallecimiento nus sorprendió tambićn a nusotros. En su última carta nos decía: «Votre Revue TBERIA est un excellent périodique, plein de choses très intéressantes. Il y a là des dessins saliniques du premier orứre. Vous savez combien j'aime l'esprit espagnol. Vous savcz aussi iout ce que j'ai fait pour sceller $k$ lieu intellectuel entre les trois nations latincs, France, Espagne, Italie. Cerre purité d'esprit s'est manifestece avant tout dans les Arts d'avant-garde où l'on n'a trouvé jusqu'ici que des artistes de cess trois pays et en tête des Atts du monde entier. Vous menez un bon combar pour l'esprit de la civilisation et je vous serre fraternellement dans mes brass (sic pour l'ensemble).

C'est ainsi qu' «beria», par le biais idéologique et guetrier, devient un porte-parole des jeunes tendances de l'avant-garde catalane et française. De son côté, J. Pérez-Jorba va s'occuper énormément de l'avant-garde françaisc et presque autant d'Apollinairs que de Pierre Albert-Birot. Je vous renvoie en ce qui concerne le premier, aux recherches de Pierre Caizergues sur «La Publicidads, et pour le sencod à l'atticle paru au numéro 4 de «Ultra», où Guillermo de Torre manifeste son admiration à l'égard d'Albert-Birot, d'Apollinaire ct de Pérez-Jorba lui-mêrme.

C'est surtout à travers les revues poćtiques, à tirage réduit et à caurte vie, que se manifeste la réception d'Apollinaire en Catalogne, comme on a vu tout à l'heure qu'il en allait pour l'Espagnc. El j'en prendrai trois comme exemple: «Troços», aUn enemic del Poble» de Salvat-Papasseit et, enfin, le cas spécial de «391\% que Picabia fait éditer à Barcelone.

Les origines de «Troģos» se situent en 1917. Peu cunnus, peu diffusée, elle n'aura pas de répercussions que dans les milieux d'avant-garde auxquels elle apports: un langage tout à fait nouveau et une mise en page aussi révolutionnairc, du point de vue poétique et plastique, que son contenu. Son orientation, à partir de la graphie, les articles, les compte-rendus, les 
traductions et les collaborateurs, nous parle constamment de l'avant-garde française. Fondée et dirigée par Junoy, elle n'aura que cirye numéros, et dans les deux derniers, c'est Foix qui la dirige et change la graphie de son titre: «Trossos». On y voit se répéter les memes noms: Cocteau, Tzara, Gleizes, Pierre et Germaine Alhert-Birot, Salmon, Dermée, Soupault. Et, dans les cinqs numćros, comme leit-motiv, Apollinaire. Il y est question de «ord-Sud" ct des propos du poère sur Baudelaire, de Calligrammes et des Mamelles à plusieurs reprises, ainsi que le avient de paraitre» de "Guynemer», ce "chef d'oeuvre des calligrammes», suivant l'expression d'Apollinaire.

De son côté, «Un enenic del Poble» paraît sn mars 1917. Le «redactor en cap» des 18 numéros sera Salvat-Papasscit er le demier numéro aura pour date celle de mais 1919. Soustitrée «Fulla de subversió espiritual», elle va de l'année III de «l'ère du crime» à el'époque de la sacrée désunions. On y trouve, parmi les signatures les plus connues, celles de Gómez de la Scrna, de lópez Picó, de "Xeniuss -Eugenio d'Ors - et Paul Dermée, Bcarcoup plus idéologique, elle est moins internationaliste. Elle a un certain contenu de classe et se veut, surtout, catalaniste. On assiste, à travers ses pages, aux débats contradictoires de Salvat-Papasseit, entre le régénctrationisme et l'avant-garde. Il se penchera enfin pour le premier non sans avoir auparavant joué un rôle essentiel darss le dévoloppement de la seconde. Plusieurs premières pages rélèvent le souci du poète pour l'écriture: la «colonne vertébrale» - «Columna vertebral: sageta de foc» - qui parait en décembre 1917, n. 9, ou bien « $54045 m_{2}$ du n. 18 , mai 1919. Mais plus intéressant encore me semble le n." 16 consacré à la mort de Folguera, en mars 1919, où les calligrammes et, en génélal, les recherches typougraphiques et synthético-idéographiques de l'écriture del Folguera occupent presque toute la prenière page pour la première fois. En y regardant de plus près, on remarque que le seul poète dont it est question -à part Folguera et le directeur de la revic - wst Apollinaire, et qu'il l'est à deux reprises. Dans la dédicace du poène de Folguera "Musics cegs de carrer»: «A Guillaume Apollinaire», mais surtout, et juste au milieu de la page, dans le «In memoriams: «De suara es en la pau del magnific Guillaume Apollinaire». En haut de la page, il y a un portrait de Folguera fait par son ami Barradas. Les paradoxes du destin feront que lorsque Barradas mourra, ce sera a son tour Guillermo de Torre qui lui fera l'éloge funèbre, qui a été publié dans \& La Gaceta Literaria» du 15 mai 1929. Cet atticle, intitulé «Adiçs a Barradas», commençait ainsi:

«Perdre

Mais perdre vraiment

pour laisser place à la trouvaille» 
On s'en doute de l'auteur. Comme quoi, Barradas a rejuint Folguera et Apollinaire dans la «paix» de ce dernier.

11 y a enfin le cas plus connu de $\$ 391 \%$, qui constitue un exemple précieux de l'accucil réservé aux avant-gardes poétiques françaises en Catalogne, et de la réception qu'à son tour trouve Apollinaire dans ces milieux. Il ne s'agit que de quatre numéros, que de seize pages, mais l'intérêt de ce qui y paraît et surtout la conception de la revuc en font un document exceptionnel.

Il est bien connu que la revue se fonde grâce a l'enthousiasme d'un certain nombre de poètes que la guerre a conduitc vers Barcelone, avec Picabia à leur tête. Dès le premier numéro, la revue essaic dc constituer une sorte de première ligne de feu de l'avant-garde pré-Dada, et situe sa rúdaction dains les déjà plusieurs foix mentionnées Galeries Dalman. Les quatre numciros «inauguraux» paraissent entre janvier et mars 1917 et soignent particulièrement les aspects poétiques et plastiques ainsi qu'une section, aOdeurs dc partout», sorte de «courrier d'informations», réelles ou factices, très bétérogène.

La personnalité de Maric Laurencin semble avoir marqué, presque autant que celle de Picabia, ces quatre numéros. A travers ses poèmes ou ses dessins, sa présence est permanente. Mais celle d'Mpollinaire, si clle cst moins explicite, n'en est pas moins éloquente. Dans le premier numéro, un dessin et un poème de $M$. Laurencin, à côte de nouvelles concernant Picassso, Max Jacob, Gleizss, parmi d'autres, ainsi que d'elle-même sous le titre «Tristouse»:

«Tristouse.-En réalité, le poèle ne fut jamais assassiné, et ce n'est pas Tristouse qui lui introduisit dans l'orbite un parapluie grand ouvert. Allez donc identifier la main...»

Au $11.0^{\circ} 3$ des textes de Ribemont-Dessaignes, de Max Jacob, plusieurs dessins de Picabia dont un intitulé «Marie». Un texte aussi de Gabriele Ruffet: «Cinématographe», illustré par un dessin de Picabia où il est écrit: «Célui qui re fait pas l'éloge du temps passé», plus, en bas, une inscription presque illisible: «... Guillaume Apollinaire» et la mention «Gloire au poète». Enfin, dans la rubrique «De nos cnvoyćs spćciauxs, à côté de nouvelles concernant Erik Satie, Picasso, Cocteau, Romain Rollatud, etc., celles de Paris, qui commencent: «Il n'est question que de Barcelone», et celles ds Barcelone où, naturellement, "Il n'est question que de Paris»:

«A peine remis de ses récents triomphes littéraires et guerricrs, verronsnous icí Guillaume Apollinaire? Plusieurs le désirent. Mais le Dieu des Armées abandonnera-t-il son lieutenant? Saint Max Jacob, pricz pour nous...» 
C'était k numéro du 1 mars 1917 . Le n. $4-25$ mars 1917 - reproduit -wpage 3 - le beau calligxamme sL'Horloge du demains, exccptionnel par les dégradés qu'on y trouve, et $\mathrm{k}$ : deux pages suivantes sont occupées par M. Laurencin, avec deux poèrnes et un dessin. Enfin, dans la dernière page de dernier numéro «inaugural», une fantaisie signée «pharamousse»: «D'une ville inforrunée». Ce sont les dernières nouvelles de «Métis-City», confuses car la censure, «empêchéc de saisir l'ésprit des correspondants» se méfie de lour écriture, «et ils échappent résolument dès qu'ils ne comprennent plus, craignant nul ne sait quois. Qui peut s'empêchcr de songer à l'kanecdotique» du poète concernant ce peintre cubiste arrêt comme espion, car ses dessins ressemblaient à des cartes secrètes? Il y a eu des élections à Métis-City. Parni les candidats, Bouvard et Pécuchet, Gleizes, Metzinger... Le lendemain: un coup d'état et voilà Picasso qui s'est couronnéc lui-même. Dans son discours, on croirait à un avant-goût de la lettrc: d'Apollinaire que Carbonell publiera lors de sa mort:

«Espagnol par mon père, italicn par ma mère et [rançais par éducation, la pureté de mes origines est le signe de ma royauté native. D'aucuns sont sacrés suthommcs, parce qu'ils ont beaucoup Iu Nietzsche et d'autres sont haussés à la dignité d'empereur qui se sont toujours montrós fervents tépublicains. Apôtre de toute liberté, j'ai pourtant reconnu l'autorité des maîres. E: je dois tour à Leonard de Vinci, Greco, Goya, les sculpteurs grees et nègres, Apollinaire, André Salmon, Max Jacob, mon marchand de coulcurs et M. Kahnweiler. Que Dieu punisse l'Institur!"

La revue «391" aura quatre numéros, avec un tirage plutôt modeste. Le dernicr numéto cotrespond au 25 mars 1917 . Entièrement écrite en français, on ne peut pas dire qu'elle ait largement contribué à diffuser Ies expériences d'avant-garde dans le public espagnol. Mais, par contre, on se doit de la considérer parmi celles qui, à un monzent donnć, ont su montrer des chemins nouveaux aux poètess espagnols, accélérant par là leur itinéraire, tout comme, d'ailleurs, celui des poètes français.

$* \quad \div \quad *$

Revenons maintenant à Junoy et à Salvat-Papasseit. Ils ont été choisis exprès et non pas au hasard, pour illustrer les coïncidences entre la postique apollinarienne et la Catalogne, pour deux raisons différentes. La première tient à ca que tous les deux dirigent une revue d'avant-garde, on a déjà vu, et, dès lors, vont se poser comme un préalable le souci de la diffusion des activités de l'avant-garde curopéene qui leur semblent les plus intéressantes.

Mais, surtout, dans la mesure où ils représentent dcux poétiques différentes, qui répondent à des prises de position divergentes et qui vont entrer 
parfois en conflict. Ce qui les unit est donc mis en valutur par ce qui les êloigne. Et ce qui les unit est justement, et au fond, l'assimilation que tous les dcux ont opérée de la poétique apollinarienne, ou, du moins d'un certain nombre de ses expériences.

Salvat-Papasseit mourra jeune, en 1924, âgé de 30 ans. Son oeuvre ćtablit les oppositions entre un futurisme poétique par lequel il se sent attiré et lc: modernisme rúǵnétrationiste et très idéologique en Catalogne, auquel il adluère. D'abord socialiste, anarchiste ensuite, il crée, âgé de 23 ans, «Un enemic del Pobles. Individualiste, épris de justice, rcjetant tout compromis, il se voit jouer le rôle prophétique et conducteur d'un «albatros». Sa première ceuvre poétique paraît en 1917: «Columna vertebral; sageta de foc», reprise dans Poomos en ondes bertzianes.

Il y joue avec la typographic, d'une façon simple mais suffisante pour définir une thématique qui est, pourtant, loin des «mots en liberté»: mćpris de la faiblesse, admiration pour la force, machinisme, lutte-énergie, jeuncssc, volonté, victoire, infini, etc., en même temps que mépris de l'expérience, de la morale, de la politique, de la philosophic, de la religion, de la raison.. Que de sophismes! Son biographe, Molas, signale que thomme fort est la «colonne vertébrale» da la société, en nême temps que la «sageta de foc» visant l'infini ${ }^{b}$.

«5-4-0-4-5» approfondir dans cette voie: recherche d'une tonalité moderne («trolley», «dynamo», etc.), désarticulation du discours, recherches typographiques faisant entrer d'autres signes què ceux spécifiquement linguistiques, etc. Il cst évident que, dans son cas, la pratique formelle tépond à un contenu thématiulue qui cherche à s'exprimer par des moyens nouveaux.

A partir de 1918, snn processus s'accélère er sc complique en raison de sa maladie et la paternité. Tour à tour, il va faire apparaître deux revues «Arc-Voltaïc» et «Proa» qui ne connaîtront qu'un seul numéro. I1 se consacre à la poésie et publie, en 1919, Poewzes en Ondes Hertzianes. L'introduction du recueil est éloquente: elle portc la signature de P. Alber-Birot: «L'art commence où finit l'imitation», suivic d'une «Lletra d'Italian, fururiste. 'Tous les concepts d'avant-garde trouvent place dans ce mélange de matériaux français ct italiens. A partir de là, il cherche souvent à s'exprimer à travers le calligramne, tout en se déclarant résolument. nationaliste et régénérationiste, et ses compositions ont parfois une nuance didactique. Nous lui devons néanmoins, même si dans ces dernières années il abandonne nonbre de ses pratiques avant-gardistes, des compositions aussi significaives que les calligrammes «Les Formigucs» ou «Romántica» qui reposent à nouveau le pro-

\footnotetext{
'Josn Salvat-Palissert: Puevies. Ed Ariel, barcelone, Classica Caralans Ariel 2, 1978, p. 8.

" JoAquin Molas, ibid., p. $x$ : "L'home forc ćs fa "columua vertebral" de la societat i, a la vegada, una "sagera de foc" que apunka al l"ufinit. Un cami que simbolitza l'oroncl!!s
} 
blème de la latéralité ou de la «plançitćs de calligranmes, comme une façon primairc de classification, par opposition, par exemple, à «Una esse», de Nogueras, ou à "Fútbol» de Carles Sindreu". Et son «XIII - El calligrama, i 2\%, paru dans El Poema de la rosa als llavis (1923) reste un singulien chef-d'oeuvre dans sa naivetê. Il devait mourir en 1924, peu après la mor: de sa fille, âgée de deux ans.

Le cas de Junoy est hien different. 'l'rès en rapport avec les Galeries Dalmau, il va très tôt deveriir un ardent diffuseur du cubisme lorsqu'il publie Arte y Artistas, en 1912. Tout ce que Salvat-Papasseir voue au nationalisme, Junoy le consacre à l'internationalisme poétique, et il établit de solides échanges entre Paris et Barcelone, en mềme temps qu'il crée son langage personnel explorant les possibilités offertes par la typographie. Il écrit en castillan jusqu'à 1916 , date à laquelle il commence à composer en catalan et à représenter, à côté de J. Pérez-Jorba, un des piliers de l'avantunarde dans sod pays. Son contact avec Apollinaire passe à travers Picrre Albert-Birot et, comme il est bien connu, il va se concrétiser dars cctte \&Carta-Prefacis à 'édiuion de Poentes $i$ Cal-ligramar, séalisćc en 1920 par Salvat-Papasseit.

Car, en effet, aussi différcntes qu'ís soient -et ils le sont-, ils coincident parfois, et même très souvent. Dans le seul numéro de «Proa», on trouve "Les Formigues» de Salvat.Papasseit, mais if y a aussi une composition aigre-douce de Junoy, "Jenny», à laccent très apollinarien.

Les audaces de Junoy se prolongent jusqu'aux années $18-20$, encorc quue ses compositions ultérieures permetten de soutenir que son retour au néoclassicisme ne le gêne pas pour incorporẹ certains procédés de l'avant-garde.

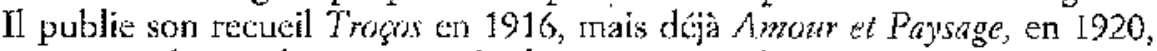
témoigne de ce tharigement de front. N'empêche que, avant cette date, Junoy avait presque crée une école d'où allait prendre son élan le poète peut-être le plus apollinairen parmi les catalans: Solé de Sojo.

Une fois marquées les différences entre les deux poètes, il cst temps de mettre aussi en valeur ce qui les unit. L'élénent commun est, d'atbord, les recherches typographiques, scripturales et plastiques. Junoy avoue lui-même sa dette, dans ce domaine, envers Apollinaire. Il y a chez lui une prise de position très nettc; en ce qui concerne les calligrammes, la typographie et

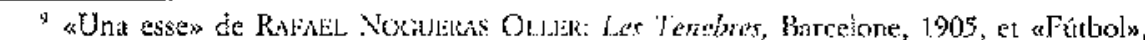

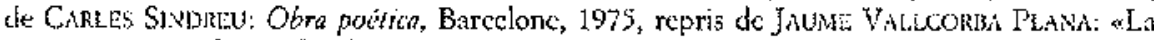

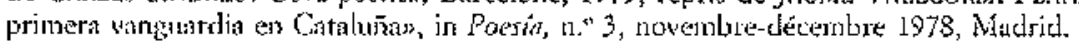


la disposition de la page, qui est plus proche d'Apollitaire que des futuristes «mots en liberté», et il suffil de contenpler «Jungleurs» ou «La Pluja de Pétals» pour s'en rendre compte ${ }^{10}$. Mais déjà la publication de Troços, le recueil poétique, suppose un événement dans le domaine de l'écriture par l'importance accordéc - et c'est la première fois que cela se manifeste d'une façon systematique-m aux éléments plastiques et visuels au détrinent des logico-discursifs. Plus tard, l'orientation plastique de la revue sera suffisamment large pour accueillir des éléments cubistes de Metzinger, des futuristes de Celso Lagar, des intetprétations métaphysiques de Miró ou, encorc, des prê-surréalistes de Ricart.

Le point de vue de Salvat-Papasseit et sa revuc sont assez différents, on l'a déjà vu. Mais la recherche d'un nouveau langage s'y fait de plus en plus déterminante, accompagnée de l'importance accordée à la visualité, d'une façon progressive, dans les illustrations des peintres espagnols. Le numéfo 9 de «Un enernic del Puble» incorporait, en première page, l'idéogramme de «Colonne vertébrale» et, à partir de là, les calligrammes vont se rendre Iréquents. I) point de vue de l'écriture, le numéro le plus important étais celui consacré à Folguera lors de sa mort. It puis, ils vont renoncer tous les deux à l'emploi systématique de l'écriture calligrammatique. Junoy, vers 1919. En ce qui concerne Salvat-Papasseit, iI en publicra deux encore dans El Pocma de la rosu als llavis. «Romântica» ne sera publiè, dans Ossa Menor, jusqu'en 1925, mais ks procédé perd de l'importance, pour lui, de jour en jour. Pour reprende un peu de la perspective, il faut rappeler qu'à la même Epoque, Finesto Caballero, Latrea, Adriano del Valle, Isaac del Vando Villar, Ramón Basterra et bien d'autres - pour ne pas citer à nouveau Guillermo de Torre, Tablada, Gómez de la Serna ou Solé de Sojom-, se livrent plus ou moins régulièrement à des recherches calligrammatiques.

Chez tous les deux, donc, même admiration et connaissance de l'oeuvre: d'ipollinaire. Même filière française dans leut approche de l'avant-garde. Même pratique typographique, iłcógrammatique, calligrammatique, à partir des exporiences apollinariennes, et peu ou rien en commun avec les «mots cn libcrés mềme si, chez les deux, à un monent donné, la thématique futuriste a été déterminante. Lit, chez tous les deux à nouvéau, il s'agit d'une période qui s'achève chez Junoy vers 1919, el, dans le cas de Salvat-Papasseit, vers 1922.

Cocí étant dit, il est évident que la recherche d'un langage suppose la rupture avec les conventions et que l'assimilation qu'ils pratiquent des formes expressives appollinariennes est éloquent à cet égard. 11 y aurait d'autres aspects à analyser. Il y a un versant thématique dont le côté «modernitér apporterait de nouvelles preuves, dans le domaine lexical par exemple. Ces

:0 J. M." JoNoy: Truģos, Leteradura, Barcelone, 1977. 
marques d'une civilisation nouveile que sont l'avion chez Junoy ou l'électricité et les tramways chez Salvat-Papasseit, on les a aupatavant recontrées dans Apollinaire. Il y aussi l'aspect idéologique, à partir du lien que l'on pourrait établir entre le régénérationisme de Salvat-..Papasseit et l'Esprit Nouveau, tel quc Margareth Wijk I'a analysé. Il y aurait encote -et ceci est bien plus délicat- à établir une étude comparée qui ne tienne compte que des aspects poétiques, qui s'intéresse au rythme, aux images, à la phonćtique, et proprement stylistique; ćtude pour laquelle on pourtait prendre pour point de départ, par excmple, "Jenny" de Junoy, mais qui poserait tous les problèmes d'une culture acquise et non innée, etc. J'ai choisi, néanmoins, de rester à ce riveau, plus froid et moins litteraire sans doute, mais qui fournit des documents précis et établit des faits concrets et réels.

Dans une certaine mesure, la boucle est fromée. On a vu lintérêt de la Catalogne: pour l'oeuvre apollinariente ct comment cet intérêt correspond à celui qui hui est voué en Castille aussi, à Madrid. On s'est attardé à ćtudier de près les moyens qui véhiculisent sa connaissance en Ispagne et comment, à travers les dates, s'établissent les traductions et les études. Mais il est certain que cet intérêt n'est pas grảuit. Il va de pair avec l'attrait pour une poétique qui assimile ses moyens expressifs et notamment la pratique calligrammatique pour exprimer une thématique nouvellc ct créer une poétique révolutiontnaire. Et cela, on l'a vu dans ces deux poc̀tes tellement éloignés, d'un point de vue idéologique, l'un de l'autre. Ce qui montre qu'il arrivait quelque chose qui les dépassait, eux deux, en tant qu'individus. Et ce qui se passe, c'est ce phénomètnc nouveau: un siècle qui cherche à se donner des signes d'identité culturelle et poétique nouvelles, par-dessus les frontières. Et, en tout cas, la figure d'Apollinaire qui rode et plâne, parfois explicite, d'autres fois larvée, mais toujouts déterminante, en Espagne. 